\title{
Chain dynamics and power-law distance fluctuations of single-molecule systems
}

\author{
Jau Tang* and R. A. Marcus ${ }^{\dagger}$ \\ Noyes Laboratory of Chemical Physics, California Institute of Technology, Pasadena, California 91125, USA
}

(Received 9 September 2005; published 23 February 2006)

\begin{abstract}
Chain-dynamics-induced distance fluctuations between any two points in a finite chain with or without cross links are investigated. This model leads to three regimes of temporal behavior for distance autocorrelation: (i) initial flat time dependence, (ii) $t^{-\alpha}$ power law, and (iii) long-time exponential decay. For an ideal Rouse chain with frequency-independent friction, $\alpha=1 / 2$. The span of the characteristic power-law behavior of a long chain could be reduced significantly with the presence of cross links.
\end{abstract}

DOI: 10.1103/PhysRevE.73.022102

Recent developments in single-molecule spectroscopy have generated wide interest in probing the conformational dynamics of a single molecule ([1], and references therein). For example, Xie and co-workers [2-5] showed that by probing the electron transfer (ET) between a donor-acceptor $(D-A)$ pair in a protein one can obtain information about distance fluctuations and protein conformational dynamics. They considered and analyzed several models, such as discrete-state hopping [3], Brownian diffusion [3], the fractional Fokker-Planck equation approach [4], and the fractional Brownian noise (FBN) model [5]. Numerous theoretical studies of reactions controlled by a fluctuating barrier have been made earlier by many including Wang and Wolynes [6], Bicout et al. [7], Bareykin et al. [8], Barkai et al. [9], and Granek and Klafter [10]. The single-molecule ET studies by Xie's group prompted us to consider the possible behavior for linear systems based on chain dynamics. The results are described in the present Brief Report.

In a recent study Granek and Klafter [10] showed that with "fractons," the fractal vibrational excitations, the distance autocorrelation function (ACF) follows power-law dependence. They noted that one-dimensional vibrational excitations could lead to temporal behavior similar to those of the FBN process [11], used by Min et al. [5] to obtain a Mittag-Leffler function [9] with a parameter $H \sim \frac{3}{4}$. Although self-similarity in fractal dynamics generally yields powerlaw behavior, a power law can also arise even in its absence. For example, the power law of fluorescence intermittency in single quantum dots can be interpreted in terms of a diffusion-controlled electron-transfer mechanism [12].

In this work, we first consider an idealized chain model to investigate distance fluctuations due to chain dynamics and later an extension to a chain with cross links. Illustrated in Fig. 1, the Rouse model, with a linear chain of beads coupled to the nearest neighbors by a spring, has been used extensively in studies of polymers ([13,14], and references therein) and more recently in an electrophoretic study of DNA [15]. Such a model is appropriate for describing a protein/polymer chain with no links between beads other than the nearest neighbors. The effects of cross links will be discussed later. Denoting $\mathbf{Q}$ as $\left(\mathbf{q}_{0}, \mathbf{q}_{1}, \mathbf{q}_{2}, \ldots, \mathbf{q}_{N-1}\right)$, a vector

\footnotetext{
*Electronic address: jautang@ caltech.edu

†Electronic address: ram@caltech.edu
}

PACS number(s): 05.40.-a, 82.37.-j, 47.53.+n, 87.15.He

with $\mathbf{q}_{k}$ representing the displacement vector for the $k$ th bead from its equilibrium position $\mathbf{q}_{k \text {,eq }}$, the Langevin equation of overdamped oscillators in an $N$-unit chain can be expressed by

$$
\zeta \frac{d}{d t} \mathbf{Q}(t)+\omega^{2} \mathbf{R} Q(t)=F(t) / m
$$

and the generalized Langevin equation is given by

$$
\int_{0}^{t} d \tau \zeta(t-\tau) \frac{d}{d \tau} \mathbf{Q}(\tau)+\omega^{2} \mathbf{R} Q(t)=F(t) / m .
$$

The Rouse coupling matrix $\mathbf{R}$ is defined as $\mathbf{R}_{i j}^{\mu \nu}=\delta_{\mu, \nu}\left(2 \delta_{i, j}\right.$ $-\delta_{i, j+1}-\delta_{i+1, j}$ ), where $\mu$ and $\nu$ represent $x, y$, or $z$, whereas $i$ and $j$ represent the bead index. At the end point $\mathbf{R}_{0 j}^{\mu \nu}$ $=\delta_{\mu, \nu}\left(\delta_{0, j}-\delta_{1, j}\right)$, and $\mathbf{R}_{i, N-1}^{\mu \nu}=\delta_{\mu, \nu}\left(\delta_{i, N-1}-\delta_{i+1, N-1}\right)$. $\mathbf{F}(\mathrm{t})$ in Eq. (1b) represents Gaussian noise with zero mean and has no correlation with $\mathbf{Q}$. Specifically, one has $\left\langle F_{i}^{\mu}(t) F_{j}^{\nu}(\tau)\right\rangle$ $=m k_{B} T \zeta(t-\tau) \delta_{i j} \delta^{\mu \nu},\left\langle F_{i}^{\mu}(t)\right\rangle=0$, and $\left\langle F_{i}^{\mu}(t) q_{j}^{\nu}(t)\right\rangle=0$. If $\zeta(t)$ $=2 \zeta \delta(t)$, Eq. $(1 \mathrm{~b})$ reduces to Eq. $(1 \mathrm{a})$.

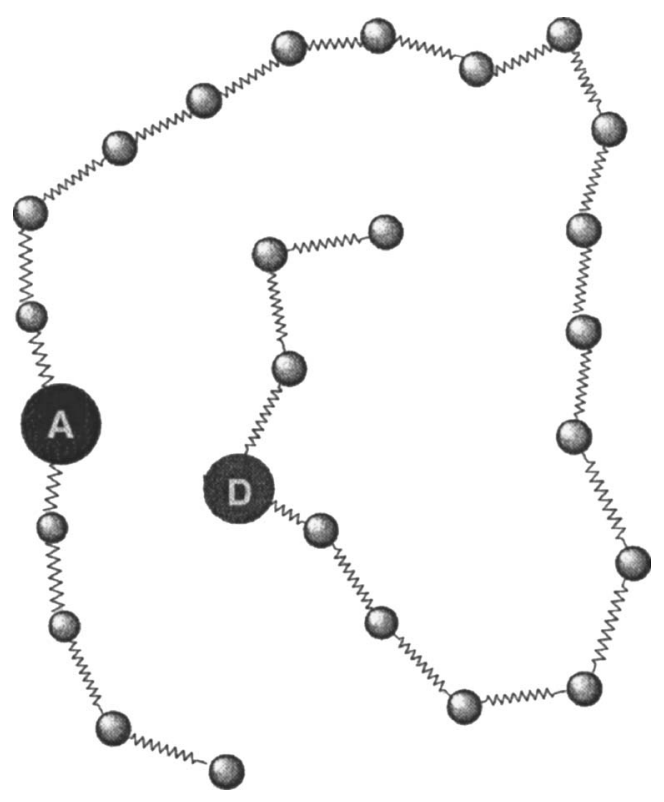

FIG. 1. Schematic diagram of a Rouse model with a chain of beads coupled by a spring to their nearest neighbors, containing a donor $(D)$ and an acceptor $(A)$. 
We first consider Eq. (1a) where $\zeta$ is a constant friction coefficient and treat Eq. (1b) later. Defining $\Gamma=4 \omega^{2} / \zeta$, one can show that the pairwise correlation of displacement vectors satisfies

$$
\begin{aligned}
& \frac{d}{d t}\left\langle\mathbf{q}_{0}(t) \cdot \mathbf{q}_{i}(0)\right\rangle+\frac{\omega^{2}}{\mathrm{~s}}\left[\left\langle\mathbf{q}_{0}(t) \cdot \mathbf{q}_{j}(0)\right\rangle-\left\langle\mathbf{q}_{1}(t) \cdot \mathbf{q}_{j}(0)\right\rangle\right]=0, \\
& \frac{d}{d t}\left\langle\mathbf{q}_{n}(t) \cdot \mathbf{q}_{j}(0)\right\rangle+\frac{\omega^{2}}{\mathrm{~s}}\left[2\left\langle\mathbf{q}_{n}(t) \cdot \mathbf{q}_{j}(0)\right\rangle-\left\langle\mathbf{q}_{n-1}(t) \cdot \mathbf{q}_{j}(0)\right\rangle\right. \\
& \left.\quad-\left\langle\mathbf{q}_{n+1}(t) \cdot \mathbf{q}_{j}(0)\right\rangle\right]=0, \\
& \frac{d}{d t}\left\langle\mathbf{q}_{N-1}(t) \cdot \mathbf{q}_{j}(0)\right\rangle+\frac{\omega^{2}}{\mathrm{~s}}\left[-\left\langle\mathbf{q}_{N-2}(t) \cdot \mathbf{q}_{j}(0)\right\rangle\right. \\
& \left.\quad+\left\langle\mathbf{q}_{N-1}(t) \cdot \mathbf{q}_{j}(0)\right\rangle\right]=0,
\end{aligned}
$$

when $n=1, N-2$ and $j=0, N-1$. Using the method described in Appendix $\mathrm{C}$ of Ref. [16] and definding $\lambda_{k}$ $=\Gamma \sin ^{2}(k \pi / 2 N),\left\langle\mathbf{q}_{i}(t) \cdot \mathbf{q}_{j}(0)\right\rangle$ is given by

$$
\begin{aligned}
\left\langle\mathbf{q}_{i}(t) \cdot \mathbf{q}_{j}(0)\right\rangle= & \frac{1}{N} \sum_{n=0}^{N-1}\left\langle\mathbf{q}_{n}(0) \cdot \mathbf{q}_{j}(0)\right\rangle+\frac{2}{N} \\
& \times \sum_{n=0}^{N-1}\left\langle\mathbf{q}_{n}(0) \cdot \mathbf{q}_{j}(0)\right\rangle \sum_{k=1}^{N-1} \cos \left[\frac{k \pi}{N}\left(i+\frac{1}{2}\right)\right] \\
& \times \cos \left[\frac{k \pi}{N}\left(n+\frac{1}{2}\right)\right] \exp \left(-\lambda_{k} t\right) \\
= & \frac{3 k_{B} T}{2 N m \omega^{2}}+\frac{3 k_{B} T}{N m \omega^{2}} \sum_{k=1}^{N-1} \cos \left[\frac{k \pi}{N}\left(i+\frac{1}{2}\right)\right] \\
& \times \cos \left[\frac{k \pi}{N}\left(j+\frac{1}{2}\right)\right] \exp \left(-\lambda_{k} t\right) .
\end{aligned}
$$

Because $\ell(t)=\left|\mathbf{q}_{n_{1}}(t)+\mathbf{q}_{n_{A}, e q}-\mathbf{q}_{n_{D}}(t)-\mathbf{q}_{n_{D}, e q}\right|$ is the distance of the $D-A$ at beam index $n_{D}$ and $n_{A}$, defining $\delta \ell(t)$ as the distance deviation from its equilibrium value $\left|\mathbf{q}_{n_{A}, e q}-\mathbf{q}_{n_{D}, e q}\right|$, the ACF $C_{Q}(t)$ can be calculated from Eq. (3) and is given by

$$
\begin{aligned}
C_{Q}(t)= & \langle\delta \ell(t) \delta \ell(0)\rangle \\
\approx & \frac{1}{3}\left\langle\left[\mathbf{q}_{n_{A}}(t)-\mathbf{q}_{n_{D}}(t)\right] \cdot\left[\mathbf{q}_{n_{A}}(0)-\mathbf{q}_{n_{D}}(0)\right]\right\rangle \\
= & C_{Q}(0) \sum_{n=1}^{N-1} \frac{1}{N}\left\{\cos \left[\frac{n \pi}{N}\left(n_{A}+\frac{1}{2}\right)\right]\right. \\
& \left.-\cos \left[\frac{n \pi}{N}\left(n_{D}+\frac{1}{2}\right)\right]\right\} \exp \left[-\Gamma t \sin ^{2}\left(\frac{n \pi}{2 N}\right)\right],
\end{aligned}
$$

where $C_{Q}(0) \equiv 2 k_{B} T / m \omega^{2}$. Equation (4) was obtained by a Taylor's series expansion of the $D-A$ distance deviation in terms of $\mathbf{q}_{k}(t)$. To analyze the fluctuations in single molecule ET rate [4,5], one is concerned about small distance changes from that of the equilibrium conformation. We assume that the ratio of $D-A$ distance fluctuations with respect to the equilibrium $D-A$ distance is small and is primarily due to stretching or contraction of the springs. If the distance fluctuation is large such as caused by rotation of a segment around a bond, higher-order terms in the expansion need to be included and Eq. (4) would become more complicated. The fluctuating $\mathbf{q}_{k}(t)$, driven by stationary white noise $\mathbf{F}(t)$, represents a Gaussian process with zero mean, $\left\langle\mathbf{q}_{k}(t)\right\rangle=0$, and all higher-order correlations can be reduced to two-time correlation pairs. In the limit of a very large $N$, Eq. (4) can be simplified to

$$
\begin{aligned}
C_{Q}(t) / C_{Q}(0)= & \int_{0}^{\pi} \frac{2 d \theta}{\pi}\left\{1-\cos \left[\left(n_{A}+n_{D}\right) \theta\right]\right\} \\
& \times\left\{1-\cos \left[\left(n_{A}-n_{D}\right) \theta\right]\right\} \exp [-\Gamma t(1-\cos \theta) / 2] \\
\approx & t^{-1 / 2} / \sqrt{\Gamma \pi}, \quad \text { if } 1 \ll \Gamma t \ll\left|n_{D}-n_{A}\right| .
\end{aligned}
$$

In some applications of the Rouse model to NMR relaxation studies of polymers [14] one is interested in the distance ACF between two ends of a chain-i.e., $n_{A}=0$ and $n_{D}=N-1$. Equation (4) yields

$$
C_{Q}(t) / C_{Q}(0)=\sum_{k=\text { odd }}^{N-1} \frac{2}{N} \cos ^{2}\left(\frac{k \pi}{N}\right) \exp \left(-\lambda_{k} t\right),
$$

and in the limit of a large $N$ it becomes

$$
C_{Q}(t) / C_{Q}(0)=\exp (-\Gamma t / 2)\left[I_{0}(\Gamma t / 2)+I_{1}(\Gamma t / 2)\right],
$$

where $I_{p}(x)$ is the modified Bessel function of the first kind of order $p$. At large $x, I_{p}(x) \sim \exp (x) / \sqrt{2 \pi x}$ [17], one has $C_{Q}(t) / C_{Q}(0) \approx t^{-1 / 2} / \sqrt{\Gamma \pi}$. Equation (7) can also be expressed by Laplace transformation [17] as

$$
\bar{C}_{Q}(s) / C_{Q}(0)=2 / \sqrt{s}(\sqrt{s+\Gamma}+\sqrt{s}) .
$$

If $\zeta$ is time dependent and has a memory, one needs to use Eq. (1b) with a time-retarded memory kernel $\zeta(\tau)$. By replacing $\Gamma$ in Eq. (8) with $\bar{\Gamma}(s)$ or $4 \omega^{2} / \bar{s}(s)$, one obtains

$$
\bar{C}_{Q}(s) / C_{Q}(0)=2 / \sqrt{s}[\sqrt{s+\bar{\Gamma}}(s)+\sqrt{s}] .
$$

If $\bar{\Gamma}(s) \propto s^{\eta}$ at small $s$, by inverse Laplace transformation one obtains an asymptote

$$
C_{Q}(t) / C_{Q}(0) \propto t^{-(1-\eta) / 2} .
$$

Such a generalization to a frequency-dependent friction leads to similar fractal behavior as recently discussed by Granek and Klafter [10]. For the special case with a constant $\zeta$, one has Eq. (6) corresponding to $\eta=0$.

If the spring constant and the friction for each chain in an ensemble are not uniform, but with a distribution-i.e., $\omega$ or $\zeta$ is distributed-we assume a normalized distribution $\tau_{c}^{2} \exp \left(-\Gamma \tau_{c}\right)$ in $\Gamma$ (or $\left.4 \omega^{2} / \zeta\right)$ where $1 / \tau_{c}$ is the characteristic distribution width. Averaging Eq. (7) over such a distribution, we obtain a very simple time dependence

$C_{Q}(t) / C_{Q}(0)=\frac{2}{1+\sqrt{1+t / \tau_{c}}}, \quad C_{Q}(0) \equiv 2 k_{B} T / m \omega_{c}^{2}$,

where $\omega_{c}^{2}=\zeta / 4 \tau_{c}$. Again, one obtains the same asymptotic $t^{-1 / 2}$ power law. If other distribution functions were used, $C_{Q}(t)$ might not be as simple and the short-time behavior could be different.

We now examine the temporal behavior of $C_{Q}(t)$ over the 
(a)

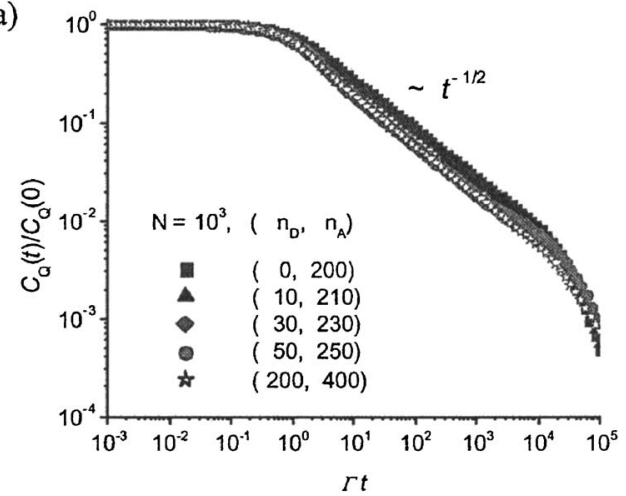

(b)

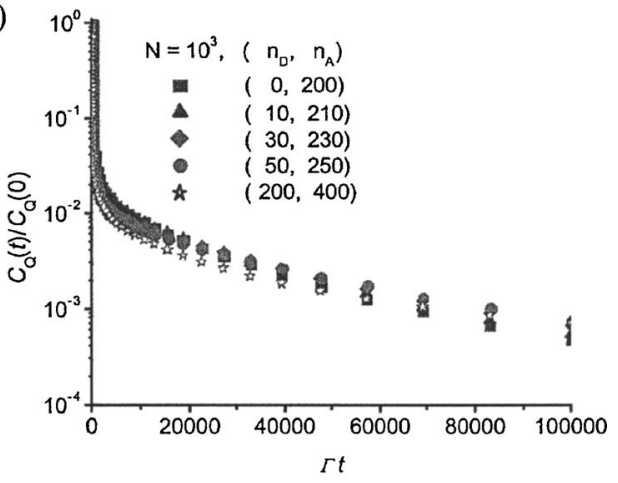

FIG. 2. (a) $C_{Q}(t) / C_{Q}(0)$ of Eq. (4) for a $D-A$ pair attached to various positions $\left(n_{D}, n_{A}\right)$. (b) Semilogarithmic plot of $C_{Q}(t) / C_{Q}(0)$ showing long-time exponential decay which is dictated by $\mid n_{D}$ $-n_{A} \mid$. The decay rate is insensitive to the attached position, and $\left|n_{D}-n_{A}\right|=200$ is used. $1 / \Gamma$ was set at 1 for all illustrations as a time unit.

entire time span for two cases, with a $D-A$ pair at the chain ends or elsewhere. As illustrated in Figs. 2 and 3, at very short times, both cases yield a flat time dependence. As time $t$ increases, $C_{Q}(t)$ follows $t^{-1 / 2}$ power-law behavior. The transition time between these two regimes is dictated by $1 / \Gamma$ and the attached positions of the $D-A$ pair. At a much later time, $C_{Q}(t)$ follows an exponential decay. The time of this change in Fig. 2 is dictated by $\left|n_{D}-n_{A}\right|^{2}$. For the chain-end attachment of Fig. 3, however, such a transition time is dictated by the Rouse relaxation time constant $T_{R}=4 N^{2} / \Gamma \pi^{2}$, which depends on $N^{2}$. In Fig. 4 we compare $C_{Q}(t)$ of Eq. (11) with Eq. (7) and with the Mittag-Leffler function of $H=3 / 4$, which is equivalent to $\exp (\Gamma t / 4) \operatorname{erfc}(\sqrt{\Gamma t / 4})$ [9]. In a more recent study, Debnath et al. [18] considered a continuous chain model, a variation of the discrete model of this work. They also concluded that a flexible chain model could reproduce the above desired value for $H=\frac{3}{4}$ as also shown independently above.

We now consider the temporal behavior according to Eq. (9) with a frequency-dependent $\bar{\Gamma}(s)$. For simplicity, assuming $\bar{\Gamma}(s)=\Gamma_{0}\left(s+\Gamma_{1}\right)^{-\eta} s^{\eta}$ so that $\bar{\Gamma}(s) \sim \Gamma_{0}$ for large $s$, and $\bar{\Gamma}(s) \sim \Gamma_{0} \Gamma_{1}^{-\eta} s^{\eta}$ for small $s, C_{Q}(t)$ of Eq. (9) can be calculated by numerical inverse Laplace transformation. As illustrated in Fig. 5(a), $C_{Q}(t)$ exhibits a $t^{-\alpha}$ power law at long times, where $\alpha=(1-\eta) / 2$. A similar power-law dependence was discussed by Granek and Klafter [10] using a different approach.
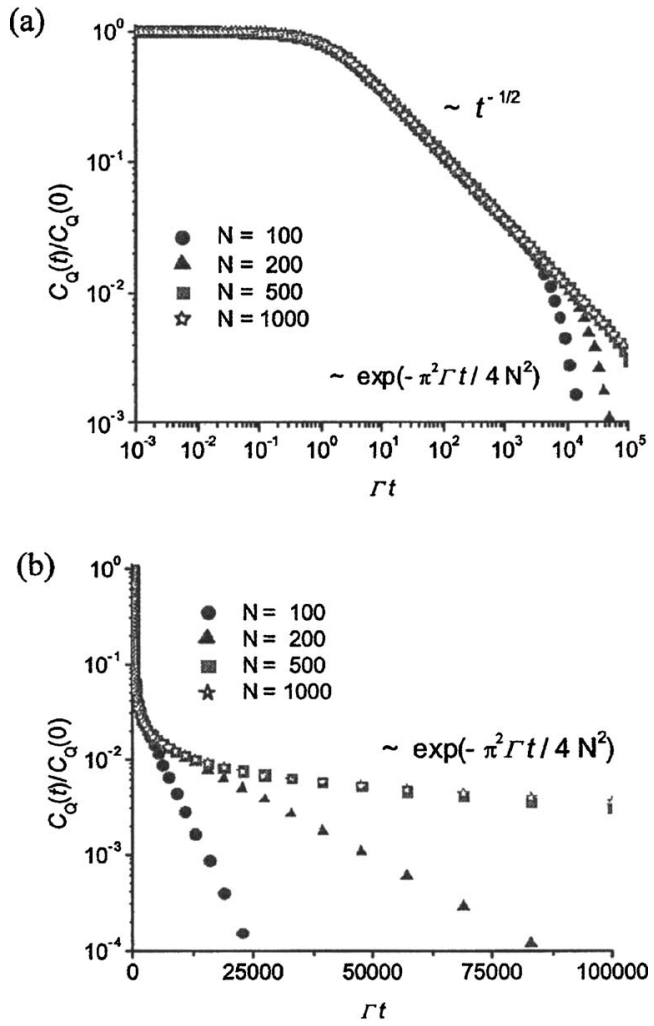

FIG. 3. (a) $C_{Q}(t) / C_{Q}(0)$ of Eq. (6) for various bead units $N$. $C_{Q}(t) / C_{Q}(0)$ stays at 1 if $\Gamma t \ll 1$, becomes a power law of $t^{-1 / 2}$ when $\Gamma t \gg 1$, and then breaks down much later. (b) Semilogarithmic plot of $C_{Q}(t) / C_{Q}(0)$ showing a single-exponential decay at long times as $\exp \left(-t / T_{D}\right)$ where $T_{D}=4 N^{2} / \Gamma \pi^{2}$.

Finally we consider the effects of cross links. With the inclusion of cross links, the coupling matrix $\mathbf{R}$ no longer possesses the simple band matrix form and numerical methods are needed to calculate the distance ACF. As illustrated in Fig. 5(b), showing deviations from the ideal Rouse model, the exponential tail becomes more prominent as the cross links increase. Because the ET rate decreases exponentially with distance, for an activationless ET to occur in $10^{-9} \mathrm{~s}$ for proteins, the separation is about $15 \AA$ [19] and is shorter if

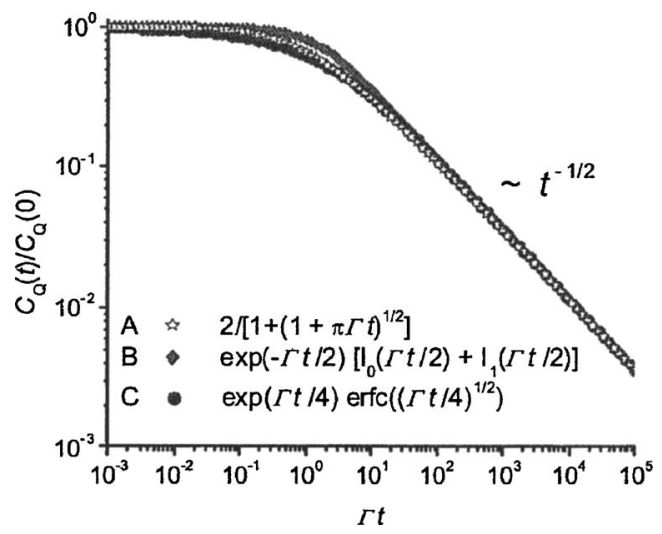

FIG. 4. Comparison of $C_{Q}(t) / C_{Q}(0)$ among the Rouse model with a distributed $\Gamma$ (curve $A$, with $\Gamma$ set at $1 / \pi \tau_{c}$ ), the ordinary Rouse mode (curve $B$ ), and the FBN model (curve $C$ ) with $\exp (\Gamma t / 4) \operatorname{erfc}(\sqrt{\Gamma t / 4})$. 
(a)

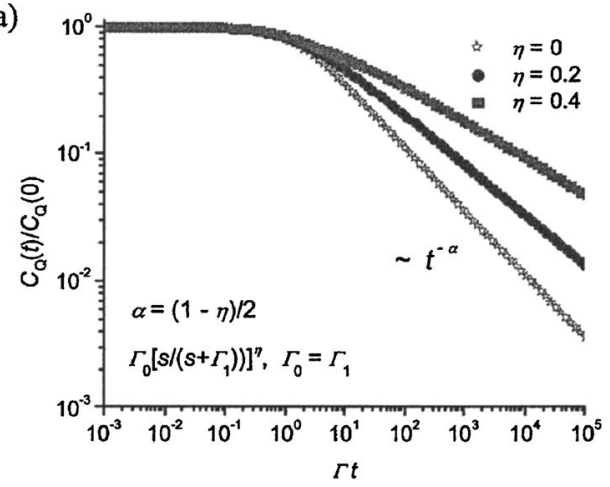

(b)

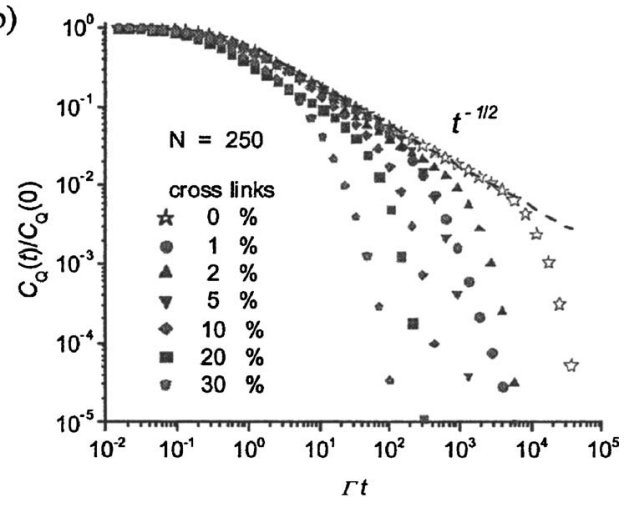

FIG. 5. (a) $C_{Q}(t) / C_{Q}(0)$ for the Rouse model with a frequencydependent friction and $\bar{\Gamma}(s)=\Gamma_{0}\left(s+\Gamma_{1}\right)^{-\eta} s^{\eta}$, following an asymptotic power law of $t^{-\alpha}$, where $\alpha=(1-\eta) / 2$. For frequencyindependent friction, $\eta=0$ and $\alpha=\frac{1}{2}$. Here $\Gamma_{0}=\Gamma_{1}=1$ was used. (b) $C_{Q}(t) / C_{Q}(0)$ for a chain of 250 units, ensemble averaged over random positions for various percentages of cross-linked beads. In addition to the nearest-neighbor couplings, a $10 \%$ of cross links, as an example, means that 25 pairs of randomly chosen beads are coupled by the harmonic spring constant.

activation energy is involved. The time span for the powerlaw $C_{Q}(t)$ depends on the bead-unit separation between the $D$-A pair. To have $C_{Q}(t)$ with a power law cover two decades

in time $[4,5]$, we estimate that more than 30 bead units between $D-A$ are needed, and so the chain would also need to loop back to bring the $D$-A within $15 \AA$ proximity or closer. We have analyzed and fitted the data of Min et al. [5] using Eq. (11), and with $\beta \sim 1.4 \AA^{-1}$ we estimated $C_{Q}(0)$ $=0.16( \pm 0.01) \AA^{2}$ and $\tau_{c}=0.11( \pm 0.01)$ s. At $k_{B} T \sim 25 \mathrm{meV}$, an effective force constant $m \omega_{c}^{2}$ between Kuhn segments is estimated to be about $5.1 \mathrm{~N} / \mathrm{m}$. However, the estimated $\zeta$ is several orders of magnitude larger than the ordinary value of $10^{13} \mathrm{~s}^{-1}$ for polymers. Although experimental power-law behavior can be reproduced by chain dynamics, a search for a different mechanism may be needed because of the possibility of numerous cross links in native proteins and the unexpectedly large $\zeta$.

In summary, we considered the distance autocorrelation function $C_{Q}(t)$ for a $D-A$ pair attached to any position in a finite linear chain. We examined its temporal behavior over the entire time span, based on an ideal Rouse model and some variations. $C_{Q}(t)$ was shown to exhibit three distinct types of temporal behavior, starting with a constant, then a power law of $t^{-\alpha}$, and finally a long-time exponential decay, where a $\alpha=\frac{1}{2}$ for an ideal Rouse chain with time-independent friction. Although we included the case of chain-end attachment, Eq. (4) for any attached location is more practical. The derived distance ACF for any two points in a chain with or without cross links could be applied to fluorescence resonance energy transfer (FRET) $[20,21]$. If $\delta \ell(t)$, the $D$ - $A$ distance fluctuation from its equilibrium value, is not small, higher-order terms in Taylor's expansion need to be included. Because of the slow $\ell^{-6}$ distance dependence for FRET efficiency, one can explore a $D-A$ pair at a much larger separation $(25-80 \AA)$ [21]. As in single-molecule ET, fluctuating FRET rates could also provide information about conformation-induced distance fluctuations.

The authors acknowledge the support of the National Science Foundation and the Office of Naval Research. J. T. also thanks the support of the James W. Glanville Foundation at the California Institute of Technology.
[1] Single Molecule Spectroscopy, edited by R. Rigler, M. Orrit, and T. Basché (Springer-Verlag, Heidelberg, 2002).

[2] H. Yang, et al., Science 302, 262 (2003).

[3] H. Yang and X. S. Xie, J. Chem. Phys. 117, 10965 (2002).

[4] S. C. Kou and X. S. Xie, Phys. Rev. Lett. 93, 180603 (2004).

[5] W. Min et al., Phys. Rev. Lett. 94, 198302 (2005).

[6] J. Wang and P. Wolynes, Chem. Phys. Lett. 212, 427 (1993); , Chem. Phys. 180, 141 (1994).

[7] D. J. Bicout, A. M. Berezhkovskii, and A. Szabo, J. Chem. Phys. 114, 2293 (2001)

[8] A. V. Barzykin, K. Seki, and M. Tachiya, J. Chem. Phys. 117, 1377 (2002).

[9] E. Barkai, R. Metzler, and J. Klafter, Phys. Rev. E 61, 132 (2000); R. Metzler, E. Barkai, and J. Klafter, Phys. Rev. Lett. 82, 3563 (1999).

[10] R. Granek and J. Klafter, Phys. Rev. Lett. 95, 098106 (2005).

[11] B. Mandelbrot and J. van Ness, SIAM Rev. 10, 422 (1968).
[12] J. Tang and R. A. Marcus, Phys. Rev. Lett. 95, 107401 (2005); J. Chem. Phys. 123, 054704 (2005), 123, 204511 (2005).

[13] M. Doi and S. F. Edwards, The Theory of Polymer Dynamics (Clarendon Press, Oxford, 1986), Chap. 4.

[14] R. Kimmich and N. Fatkullin, Adv. Polym. Sci. 170, 1 (2004).

[15] S. Ferree and H. W. Blanch, Biophys. J. 87, 468 (2004).

[16] J. Tang, S. N. Dikshit, and J. R. Norris, J. Chem. Phys. 103, 2873 (1995).

[17] The Transforms and Applications Handbook, edited by A. D. Poularikas (CRC Press, Boca Raton, FL, 1996).

[18] P. Debnath et al., J. Chem. Phys. 123, 204903 (2005).

[19] H. B. Gray and J. R. Winkler, Proc. Natl. Acad. Sci. U.S.A. 102, 3534 (2005).

[20] M. A. Morgan et al., Biophys. J. 89, 2588 (2005).

[21] H. Neuweiler and M. Sauer, Curr. Pharm. Biotechnol. 5, 285 (2004). 